\title{
Tuning Advanced LIGO to kilohertz signals from neutron-star collisions
}

\author{
Dhruva Ganapathy®, ${ }^{1, *}$ Lee McCuller $\odot,{ }^{1}$ Jameson Graef Rollins $\odot,{ }^{2}$ Evan D. Hallø, ${ }^{1}$ \\ Lisa Barsotti@, ${ }^{1}$ and Matthew Evans $\oplus^{1}$ \\ ${ }^{1}$ LIGO Laboratory, Massachusetts Institute of Technology, \\ 185 Albany Street, Cambridge, Massachusetts 02139, USA \\ ${ }^{2}$ LIGO Laboratory, California Institute of Technology, 1200 E California Blvd, \\ Pasadena, California 91125, USA
}

(Received 29 October 2020; accepted 17 December 2020; published 11 January 2021)

\begin{abstract}
Gravitational waves produced at kilohertz frequencies in the aftermath of a neutron star collision can shed light on the behavior of matter at extreme temperatures and densities that are inaccessible to laboratory experiments. Gravitational-wave interferometers are limited by quantum noise at these frequencies but can be tuned via their optical configuration to maximize the probability of postmerger signal detection. We compare two such tuning strategies to turn Advanced LIGO into a postmerger-focused instrument: first, a wideband tuning that enhances the instrument's signal-to-noise ratio $40-80 \%$ broadly above $1 \mathrm{kHz}$ relative to the baseline, with a modest sensitivity penalty at lower frequencies; second, a "detuned" configuration that provides even more enhancement than the wideband tuning, but over only a narrow frequency band and at the expense of substantially worse quantum noise performance elsewhere. With an optimistic accounting for instrument loss and uncertainty in postmerger parameters, the detuned instrument has a $\lesssim 40 \%$ sensitivity improvement compared to the wideband instrument.
\end{abstract}

DOI: 10.1103/PhysRevD.103.022002

\section{INTRODUCTION}

The discovery of a binary neutron star (BNS) merger by LIGO and Virgo in 2017 (GW170817) [1], and the electromagnetic followup observations of a kilonova [2], have heralded a new era of observational neutron star physics. Information about the tidal deformability of the constituent objects [3] is encoded in the gravitational waveforms of binary mergers, both in the inspiral phase before coalescence [4], and in the postmerger phase promptly thereafter [5].

While the inspiral effects occur primarily below $1 \mathrm{kHz}$, the postmerger signal is expected at kilohertz frequencies $[6,7]$. Understanding the postmerger physics therefore requires improving or targeting detector sensitivity at these higher frequencies. In particular, postmerger waveforms have been simulated for various models of the neutron star equation of state (EoS) and their Fourier spectra typically show a narrow band of signal energy concentrated around $2 \mathrm{kHz}$ [5].

In a scenario where multiple gravitational-wave detectors are operational, it may be beneficial to maximize one or more detectors for sensitivity to these BNS postmerger signals, while relying on other detectors in the network for inspiral detection and source localization. Optimizing detectors at high frequencies has been investigated in

\footnotetext{
*dhruva96@mit.edu
}

the context of future major upgrades in current and new facilities $[8,9]$, and in a proposal for a new dedicated high-frequency gravitational-wave interferometer [10]. Here we quantify the sensitivity to high-frequency, narrowband postmerger signals for modified "tunings" of the LIGO interferometers and their upcoming "A+" upgrade $[11,12]$.

Two modifications are considered, with their strain spectra densities shown in Fig. 1. The first is the "wideband" configuration, where the interferometer bandwidth is increased to encompass the expected postmerger resonances, and the second is the "detuned" configuration, where the $\mathrm{A}+$ interferometer is operated with a high-frequency, narrow-band dip. The only physical changes to the optical system associated with these new configurations are the transmissivity of the LIGO signal recycling mirror and filter cavity input mirror. Neither of these new configurations requires modifying the facility, vacuum envelope or suspension design, so either could be readily adopted as a near-term modification to an A+ LIGO interferometer.

\section{INTERFEROMETER CONFIGURATIONS}

The sensitivity of existing gravitational-wave interferometers at frequencies above a few hundred hertz is limited almost exclusively by quantum shot noise $[13,14]$. Quantum shot noise can be reduced by increasing power in the arms of the interferometer [15], injecting squeezed vacuum states into the output port [16], and by trading 


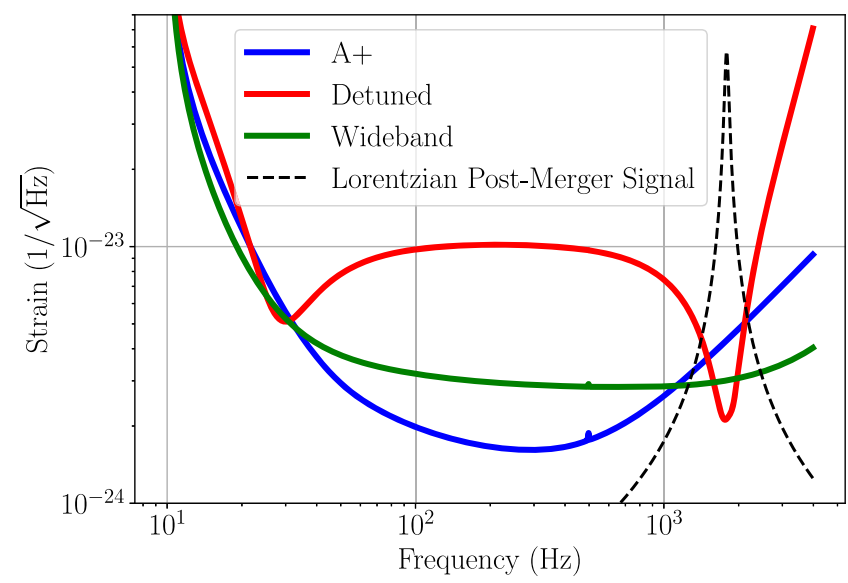

FIG. 1. Interferometer configurations under comparison. Representative strain noise curves of the "A+", detuned, and wideband configurations are plotted for reference. Both altered configurations sacrifice sensitivity at low frequencies in order to increase high-frequency sensitivity. The sensitivity improvement for the detuned configuration is across a relatively narrow band, and is achieved by detuning the signal recycling cavity in order to obtain a high-frequency resonant enhancement. In the wideband configuration, the input transmission of the signal recycling cavity is reduced in order to increase the interferometer bandwidth. The dashed black curve corresponds to the strain $\tilde{h}_{\mathrm{DS}}(f)$ of a Lorentzian postmerger signal (Eq. (1)) with $f_{0}=1798 \mathrm{~Hz}$ and $Q=28.32$, observed at $100 \mathrm{Mpc}$ with $0.1 M_{\odot}$ converted to gravitational wave energy during postmerger [see Eq. (A6)]. It is plotted in spectral density units using the form $2 \sqrt{f}\left|\tilde{h}_{\mathrm{DS}}(f)\right|$. Using Eq. (3), the signal-to-noise ratio is calculated to be around 3 for the baseline A+ configuration.

sensitivity at some frequencies for others by changing the optical parameters of the interferometer [17].

The LIGO detectors [14] use arm cavities to both increase the arm power and shape the interferometer frequency response, with the addition of a signal recycling mirror (SRM) to implement the "resonant sideband extraction" scheme [18]. The SRM forms a signal recycling cavity (SRC) that determines the detector bandwidth. In the baseline A+ configuration the SRC is operated to resonantly couple the signal out of the arm cavities, broadening the bandwidth of the detector from $40 \mathrm{~Hz}$ to $450 \mathrm{~Hz}$.

The parameters of the $\mathrm{A}+$ design, shown in Table I, are optimized for detecting inspiral signals, with quantum noise and classical thermal noise similarly affecting the detection range. The detector bandwidth, adjusted by the SRM transmissivity, is chosen to balance the peak sensitivity determined by shot noise and the degradation at low frequencies caused by radiation pressure noise. Frequencydependent squeezing $[15,19,20]$ is employed to enhance the interferometer sensitivity at all frequencies. For the various configurations described, an injected squeezing of $12 \mathrm{~dB}$ is assumed. Taking into account the injection and readout losses, which are assumed to be 5\% and 10\% respectively, the effective broadband quantum noise
TABLE I. Parameters of LIGO configurations. $T_{\mathrm{SRM}}$ and $T_{\mathrm{FC}}$ are the signal recycling mirror and filter cavity input mirror transmissions respectively, while $\phi_{\mathrm{SRC}}$ and $\Delta \omega_{\mathrm{FC}}$ are the SRC and filter cavity detuning in units of phase and frequency respectively.

\begin{tabular}{|c|c|c|c|}
\hline Parameter & \multicolumn{3}{|c|}{ Value } \\
\hline Arm power & \multicolumn{3}{|c|}{$750 \mathrm{~kW}$} \\
\hline Power on beam-splitter & \multicolumn{3}{|c|}{$5.4 \mathrm{~kW}$} \\
\hline Classical noises & \multicolumn{3}{|c|}{ Thermal noise [21] } \\
\hline SRC length & \multicolumn{3}{|c|}{$55 \mathrm{~m}$} \\
\hline $\operatorname{SRC} \operatorname{loss}\left(\Lambda_{\mathrm{SRC}}\right)$ & \multicolumn{3}{|c|}{$0.1 \%$} \\
\hline Injected squeezing & \multicolumn{3}{|c|}{$12 \mathrm{~dB}$} \\
\hline Injection loss & \multicolumn{3}{|c|}{$5 \%$} \\
\hline Readout loss & \multicolumn{3}{|c|}{$10 \%$} \\
\hline Filter cavity length & \multicolumn{3}{|c|}{$300 \mathrm{~m}$} \\
\hline Filter cavity loss & \multicolumn{3}{|c|}{$60 \mathrm{ppm}$} \\
\hline & $\mathrm{A}+$ & Wideband & Detuned \\
\hline SRM transmission $\left(T_{\mathrm{SRM}}\right)$ & 0.325 & 0.05 & Table II \\
\hline SRC detuning $\left(\phi_{\mathrm{SRC}}\right)$ & $0^{\circ}$ & $0^{\circ}$ & Table II \\
\hline Signal $3 \mathrm{~dB}$ bandwidth & $450 \mathrm{~Hz}$ & $4.8 \mathrm{kHz}$ & Fig. 2 \\
\hline Filter cavity transmission $\left(T_{\mathrm{FC}}\right)$ & 0.0012 & 0.0004 & Table II \\
\hline Filter cavity detuning $\left(\Delta \omega_{\mathrm{FC}}\right)$ & $46 \mathrm{~Hz}$ & $16 \mathrm{~Hz}$ & Table II \\
\hline
\end{tabular}

suppression in $\mathrm{A}+$ is estimated to be around $7 \mathrm{~dB}$. As radiation pressure noise is not an observable effect at the frequencies of interest for a postmerger, the relative phase between the squeezed field and the main interferometer field has been chosen to minimize shot noise.

Representative strain noise curves for the wideband and detuned configurations described below are shown along with the A+ curve in Fig. 1.

Notably, squeezing enhancement plays a crucial role when comparing these alternative configurations. At postmerger signal frequencies of $\sim 2 \mathrm{kHz}$, squeezed vacuum states are temporarily stored in the signal recycling cavity, experiencing its roundtrip loss, $\Lambda_{\mathrm{SRC}}$, repeatedly over multiple traversals. For $\mathrm{A}+$, this amounts to a loss of $\sim 10 \Lambda_{\text {SRC }}$. The wideband and detuned configurations change the storage time of the signal recycling cavity, which can result in strongly degraded squeezing as the SRC loss becomes comparable to other loss in the system.

\section{A. Wideband}

The wideband configuration increases the bandwidth of a LIGO interferometer by further reducing the SRM transmissivity. We consider $T_{\mathrm{SRM}}=0.05$, reducing the peak strain sensitivity, but extending the bandwidth beyond $3 \mathrm{kHz}$. This value is chosen so that the interferometer is sensitive to a wide range of frequencies where BNS postmerger signals are expected to lie. This configuration is not optimized for any particular postmerger model, so it is effective for detecting a variety of signals.

The decrease in peak sensitivity additionally reduces quantum radiation-pressure noise, requiring the filter cavity 
bandwidth to be decreased. This only affects sensitivity below $100 \mathrm{~Hz}$ and is not important for the analysis of postmerger signals. Similarly to A + , squeezing provides a broadband enhancement to the wideband configuration. Because the arms and SRC stay on resonance, the wideband configuration adds no additional frequency dependence to squeezing; however, decreasing $T_{\mathrm{SRM}}$ modifies how the loss $\Lambda_{\mathrm{SRC}}$ limits the squeezing enhancement.

In the wideband configuration, the loss added by the interferometer becomes $\sim 20 \Lambda_{\mathrm{SRC}}$ to $\sim 40 \Lambda_{\mathrm{SRC}}$, increasing for signals approaching the detector bandwidth. The loss changes with frequency as the squeezing field transitions from being stored in the arms to being stored in the SRC, and the increased loss is due to the lower SRM transmissivity and correspondingly longer storage time. Even so, this increased loss is still subdominant to the input and output path losses, so squeezing performance is similar between the $\mathrm{A}+$ and wideband configurations.

\section{B. Detuned}

The SRC can alternatively be operated in a "detuned" state, where it is held slightly off of resonance by maintaining an optical phase shift $\phi_{\text {SRC }}$ using feedback control. In this state, the interferometer optical response forms a resonant peak, resulting in a dip in the quantum noise spectrum in units of strain. This increases sensitivity at high frequencies at the expense of sensitivity at lower frequencies $[14,22,23]$. When the detuning is optimized for resonances in the kilohertz region, an additional narrowband optomechanical spring resonance is formed at low frequencies $(10-30 \mathrm{~Hz})$, but overall, this configuration is substantially less sensitive for inspiral detection and source localization.

The choice of detuning phase $\phi_{\text {SRC }}$ affects the frequency of the quantum noise dip; additionally, the transmissivity of the SRM narrows the resonance of the signal response and correspondingly deepens the dip in the noise spectrum. In the detuned configuration, $T_{\mathrm{SRM}}$ and $\phi_{\mathrm{SRC}}$ must be optimized to achieve maximum signal-to-noise ratio (SNR) given a distribution of center frequencies and signal bandwidths for postmerger signals. The configuration will depend on the particular postmerger model and the performance is computed for several parameter distributions which are described in Appendix.

Balanced homodyne readout of the gravitational-wave signal is proposed for A+ as an improvement over LIGO's current fringe-offset readout $[24,25]$. For the detuned case, the interferometer signal sidebands are strongly imbalanced above and below the laser frequency at the resonant dip, so there is not a preferred readout angle for the postmerger signal detection. Varying the readout angle does not significantly improve the results or impact the discussion for postmerger signals, and does not improve low frequency sensitivity, for the detuned case, beyond what is shown in Fig. 1.
The detuned configuration considerably affects squeezing in two ways. First, the unbalanced optical response of the interferometer results in a frequency-dependent rotation of the phase of the squeezed field relative to the main interferometer field, which must be compensated using a similarly unbalanced filter cavity. Along with the SRC parameters, this analysis optimizes the filter cavity input mirror transmission and resonance frequency $\Delta \omega_{\mathrm{FC}}$ to maximize average SNR [see Eq. (4)] for each parameter model. The filter cavity roundtrip loss is kept constant at the expected $\mathrm{A}+$ level of $60 \mathrm{ppm}$, which is close to the value already achieved at TAMA [26].

Second, for the parameters of Table I, the lower SRM transmissivity required for detuning causes the interferometer to inflict a squeezing loss of $\sim 200 \Lambda_{\mathrm{SRC}}$ within the narrow frequency band of the optical resonance. This loss is equal or greater than the expected total input and output losses, which prevents squeezing from providing as large a benefit to the peak strain sensitivity in the detuned case as for the wideband or A+ cases, even with optimized filter cavity parameters. Instead, the frequency dependence of the effective loss outside the interferometer bandwidth causes the squeezing to increase the effective band of the dip in strain spectral noise density. This effect is shown in the noise curves in Fig. 2, where optimized configurations with and without squeezing have been plotted together.

\section{BINARY NEUTRON-STAR POSTMERGER TEMPLATES}

Binary neutron star postmerger waveform models consistently show that much of their gravitational strain signal energy is contained within a limited frequency band [5]. For the purpose of comparing the SNR of detections, we approximate each postmerger narrowband signal as a damped sinusoid (DS) [27], which has a frequency-domain representation that is the symmetric composition of positive and negative frequency complex Lorentzian damped envelopes (DE):

$$
\begin{gathered}
\tilde{h}_{\mathrm{DS}}(f)=\tilde{h}_{\mathrm{DE}}(f)+\tilde{h}_{\mathrm{DE}}^{*}(-f), \\
\tilde{h}_{\mathrm{DE}}(f)=\sqrt{\frac{H}{4 \pi}} \cdot \frac{e^{i \theta(f)} \sqrt{f_{0} / Q}}{f_{0} / 2 Q+i\left(f-f_{0}\right)} .
\end{gathered}
$$

Here $f_{0}$ is the signal's central frequency, and its bandwidth is set by its $Q$ factor. $H$ is the total energy of the strain signal. $e^{i \theta(f)}$ indicates additional parameters in the phase response [27], but these do not affect the SNR calculations, which rely only on the magnitude of the frequency-domain signal. For the detuned configuration, the interferometer's optical resonance bandwidth and dip frequency produces the greatest SNR when it is well matched to the waveform bandwidth and center frequency, but due to loss, the interferometer dip is generally of lower $Q$ than the templates. 

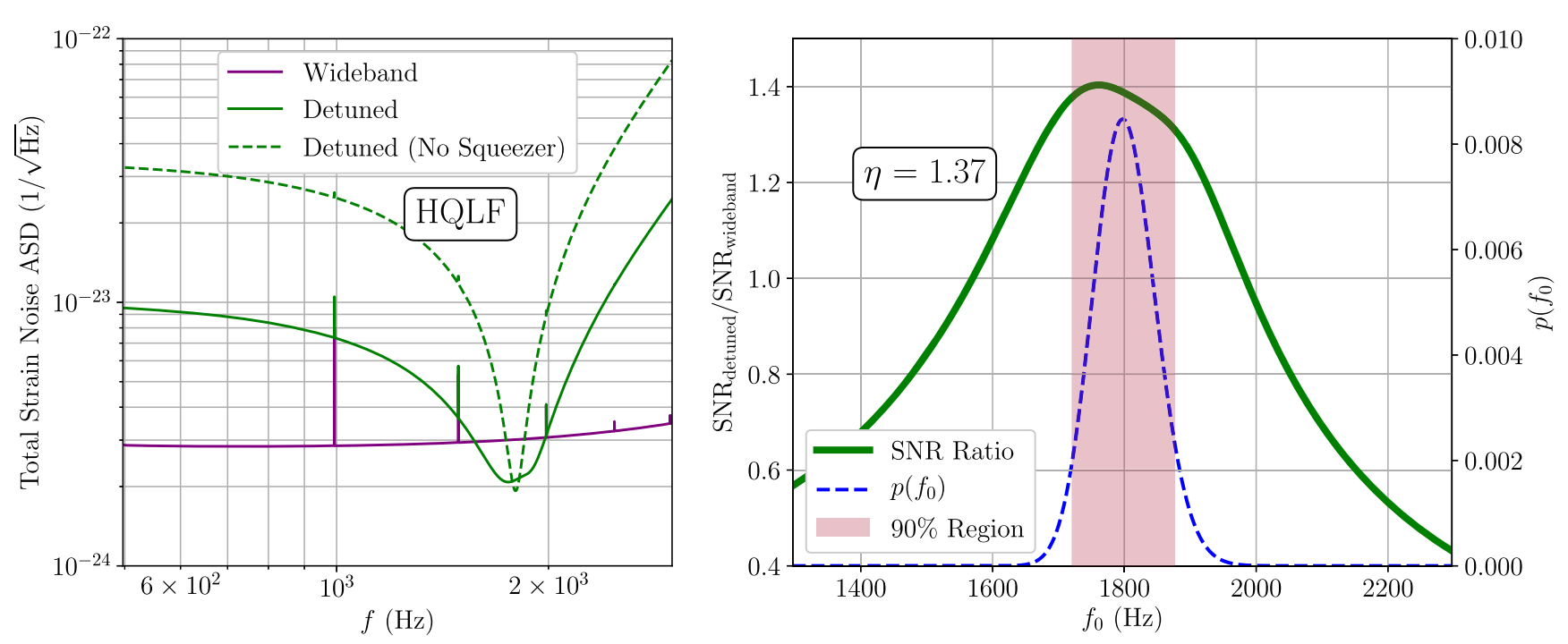

FIG. 2. Performance comparison between the detuned and wideband configurations for the high- $Q$-low-frequency (HQLF) distribution of BNS postmerger signals (Table III) which peaks at $f_{0}=1.8 \mathrm{kHz}$, and has $Q=60$. The left plot shows the strain noise curves for wideband, detuned and purely detuned (without squeezing) configurations. For the detuned configurations, the enhancement provided by squeezing in the resonant dip is degraded due to the roundtrip loss of the SRC. However, the width of the dip is broadened significantly. The plot on the right shows the signal-to-noise ratio (green trace) of the detuned configuration with respect to the wideband configuration over a range of Lorentzian central frequencies. The dashed blue trace corresponds to the probability distribution of signals as a function of central frequency. The detuned interferometer has been optimized to maximize $\eta$ for the distribution HQLF. The parameters of the detuned configuration are given in the third row of Table II. The shaded magenta region corresponds to the region containing $90 \%$ of the signal probability. The overall SNR improvement $\eta$ is calculated to be 1.37 for this configuration.

The ability to match the detector to the signals is limited by the natural variability in the center frequency of postmerger waveforms. Simulations of neutron star inspiral models have informed phenomenological relations between astrophysical system parameters and parameters of the resulting postmerger signal [27,28]. These relations lead to a varying waveforms with distribution function $p\left(f_{0}\right)$, resulting from the distribution of binary neutron star systems. Table II shows seven such distributions and their associated optimized interferometer parameters. These distributions, derived in Appendix, are not tied to specific neutron star models, but instead span the uncertainty of the phenomenological waveform parametrizations.

The SNR of each signal is calculated from each configuration's power spectral density (PSD) and the signal's waveform, $\tilde{h}_{\mathrm{DS}}(f)$, using

$$
\mathrm{SNR}_{\text {config }}^{2}=4 \int_{0}^{\infty} \frac{\left|\tilde{h}_{\mathrm{DS}}(f)\right|^{2}}{\operatorname{PSD}_{\text {config }}(f)} d f,
$$

where $\tilde{h}_{\mathrm{DS}}$ depends on the waveform's central frequency $f_{0}$ and its $Q$ factor. The average ratio of the detuned SNR to the wideband SNR, weighted over the distribution $p\left(f_{0}, Q\right)$, provides a figure of merit, $\eta$, to compare configurations:

$$
\eta^{2} \equiv \int p\left(f_{0}, Q\right) \frac{\mathrm{SNR}_{\text {detuned }}^{2}}{\mathrm{SNR}_{\text {wideband }}^{2}} d f_{0} d Q .
$$

Similarly, the efficacy of the wideband configuration compared to the baseline of $\mathrm{A}+$ is expressed as

$$
\eta_{\mathrm{WB} / \mathrm{A}+}^{2} \equiv \int p\left(f_{0}, Q\right) \frac{\mathrm{SNR}_{\mathrm{wideband}}^{2}}{\mathrm{SNR}_{\mathrm{A}+}^{2}} d f_{0} d Q
$$

TABLE II. Optimal interferometer configurations and improvement factors, $\eta$ [see Eq. (4)] for various astrophysical distributions, which are described in Table III. The SRC is $55 \mathrm{~m}$ long with a roundtrip loss $\Lambda_{\mathrm{SRC}}$ of $0.1 \%$. The filter cavity is $300 \mathrm{~m}$ long with a roundtrip loss of $60 \mathrm{ppm}$. $\eta_{\mathrm{WB} / \mathrm{A}+}$ [see Eq. (5)] shows the average SNR improvement provided by the wideband configuration with respect to $\mathrm{A}+$. The last column $f_{\text {peak }}$ is the frequency (in $\mathrm{Hz}$ ) that corresponds to the peak sensitivity in the detuning dip.

\begin{tabular}{lccccccc}
\hline \hline Dist. & $T_{\mathrm{SRM}}$ & $\phi_{\mathrm{SRC}}$ & $T_{\mathrm{FC}}$ & $\Delta \omega_{\mathrm{FC}}(\mathrm{Hz})$ & $\eta$ & $\eta_{\mathrm{WB} / \mathrm{A}+}$ & $f_{\text {peak }}$ \\
\hline LQLF & $0.72 \%$ & $2.38^{\circ}$ & $0.30 \%$ & 1843 & 1.29 & 1.38 & 1741 \\
MQLF & $0.70 \%$ & $2.36^{\circ}$ & $0.29 \%$ & 1846 & 1.34 & 1.41 & 1748 \\
HQLF & $0.69 \%$ & $2.36^{\circ}$ & $0.28 \%$ & 1850 & 1.37 & 1.42 & 1753 \\
LQMF & $0.83 \%$ & $1.37^{\circ}$ & $0.62 \%$ & 2766 & 1.04 & 1.76 & 2505 \\
MQMF & $0.78 \%$ & $1.37^{\circ}$ & $0.59 \%$ & 2765 & 1.07 & 1.81 & 2519 \\
HQMF & $0.76 \%$ & $1.36^{\circ}$ & $0.56 \%$ & 2764 & 1.09 & 1.83 & 2527 \\
HQHF & $1.13 \%$ & $0.76^{\circ}$ & $1.04 \%$ & 3783 & 1.00 & 2.10 & 3227 \\
\hline \hline
\end{tabular}



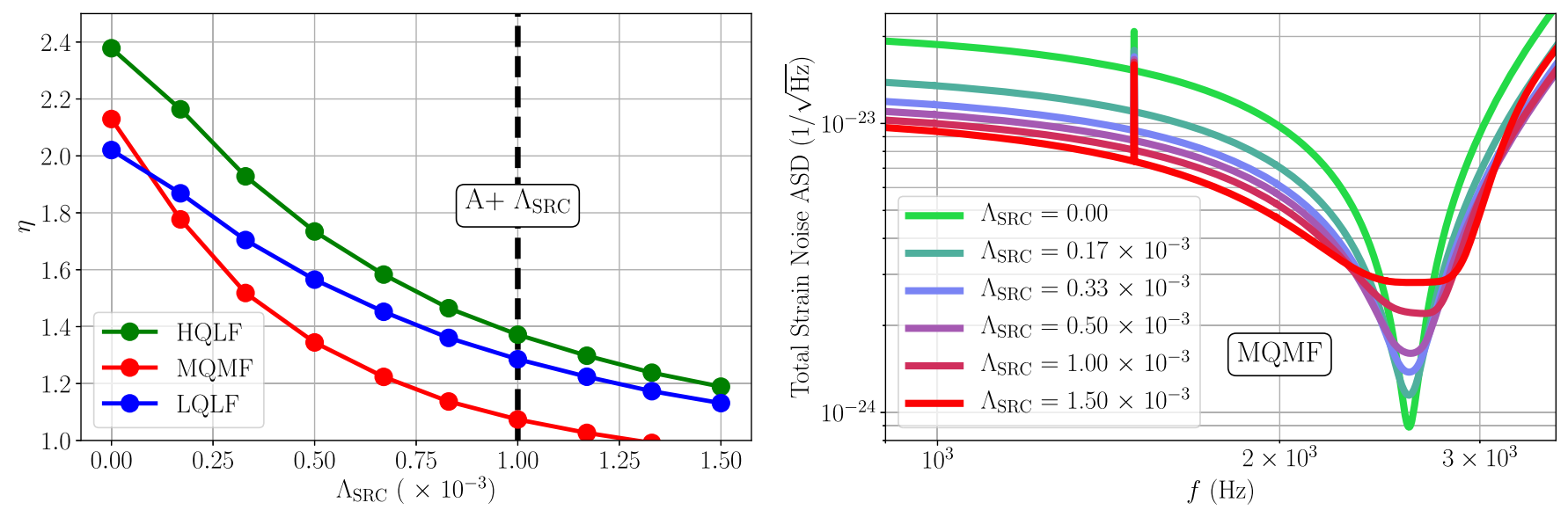

FIG. 3. The effect of SRC loss on BNS postmerger sensitivity. The plot on the left demonstrates how the relative improvement factor for optimized interferometers (Eq. (4)) is limited by the roundtrip SRC loss $\Lambda_{\mathrm{SRC}}$ for various distribution models used for interferometer optimization. The A+ SRC loss limits sensitivity improvement to around 50\% of the zero loss case. The right plot shows optimum strain curves for distribution model MQMF as SRC loss $\Lambda_{\mathrm{SRC}}$ is varied. Loss lowers the $\mathrm{Q}$ of the resonant band and squeezing widens the band to create a flat response. To maintain optimal performance, the resonance and squeezing effects of loss on dip bandwidth are balanced using the SRM transmission.

\section{RESULTS}

Table II shows the optimal interferometer parameters and relative improvement that is achieved by detuning the SRC for various astrophysical distributions of BNS postmerger signals. The wideband configuration provides an average SNR improvement $\eta_{\mathrm{WB} / \mathrm{A}+}$ of $1.38-2.10$ over $\mathrm{A}+$. The average SNR improvement from detuning, $\eta$, lies between 1.00 and 1.37 relative to the wideband for the optical parameters of Table I.

The improvement provided by detuning the interferometer is generally lower for distributions that center around higher frequencies and lower $Q$ factors. The case in which detuning is most favorable, corresponding to the distribution labelled HQLF (high-Q low-frequency), is presented in Fig. 2, which shows strain noises and the SNR improvement over a range of Lorentzian signals. This configuration has an $\eta=1.37$.

From the shaded magenta region in Fig. 2, which encloses $90 \%$ of the signals under the HQLF model, the detector dip is sufficiently wide to provide a benefit over the entire range of expected parameters. This is notable as the astrophysical distribution of the center frequencies has a spread of approximately $200 \mathrm{~Hz}$, which makes the distribution cover a wider band than the templates themselves for $Q>10$. Because of this spread, even if the interferometer were lower loss and could obtain higher peak strain sensitivity in the detuned configuration, it cannot be configured to optimally match the interferometer resonance to the template resonance due to the distribution of center frequencies. Instead, loss widens the sensitive band to cover the distribution of templates, but reduces the peak sensitivity and relative SNR improvement.

The relative benefit for the detuned interferometer, reported independently for each model distribution, provides a best-case analysis where the astrophysical model of BNS postmergers is assumed to be sufficiently constrained to allow interferometer optimization. The relative SNR can be cubed to represent the relative improvement to the detection volume or, relatedly, the relative rate of detections. The largest $\eta=1.37$ corresponds to a factor of 2.57 increase in postmerger signal detection rate over the wideband configuration. If the postmerger model is not known, the detuning center frequency must be scanned by tuning the SRC roundtrip phase, distributing time among potential detection frequencies. Scanning can thus significantly reduce the rate benefit of detuning. The wideband optimization has improved sensitivity at all of the potential models and avoids the need for scanning.

\section{ADDITIONAL CONSIDERATIONS}

\section{A. Loss in the signal recycling cavity}

Figure 3 shows the effect of SRC loss on the relative performance improvement of the detuned configuration over the wideband configuration. This figure indicates how severely the SRC detuning method is limited by optical loss within the SRC. The decreased SRM transmissivity required for a narrowband response creates an optical cavity where signal field crosses several optic surfaces and substrates such as the beamsplitter many more times than in the $\mathrm{A}+$ or wideband configurations. Because of its use of optical resonance, the detuned configuration can nearly saturate the sensitivity available given the loss [29], and squeezing tends to simply increase the bandwidth at peak sensitivity, as shown in the strain curve in Fig. 2. On the other hand, because of squeezing, the wideband configuration also approaches the maximum possible sensitivity, given loss, without sacrificing signal bandwidth. 
A roundtrip power loss of $0.1 \%$ in the SRC is used for the interferometer models in this analysis. This value is optimistic and results from adding assumed losses from all antireflection (AR) coating transmissions (500 ppm), reflections $(200 \mathrm{ppm})$, clipping on the beam splitter and other optics $(100 \mathrm{ppm})$, and imperfect interference at the beam splitter (200 ppm). Current measurements establish an upper bound $0.3 \%$ loss for LIGO interferometers in the third observing run $(\mathrm{O} 3)$.

The A+ upgrade intends to address issues that impact loss, but is unlikely to drive the SRC loss below the optimistic value used herein, reiterating that the relative improvements quoted for detuning represent best-case scenarios. Further improvements, or future detectors, may achieve lower loss in the SRC by reducing the number of AR-coating transmissions (e.g., by flipping the beam splitter to favor the SRC and removing compensation plates), reducing the number of reflections (e.g., by avoiding telescope optics in the SRC), and by reducing wavefront phase distortion (e.g., better compensating substrate index variations during polishing).

\section{B. Operational challenges}

Both the wideband and detuned configurations require lowering the SRM transmissivity from $T_{\mathrm{SRM}} \approx 30 \%$, which will alter the operating parameters of the interferometer and require time to implement. In addition to the signal fields, the interferometers also employ radio-frequency fields to sense internal degrees of freedom related to the power and signal recycling cavities, as well as the alignment of optics. The wideband configuration maintains the same operating modes for these fields and cavities, adjusted only by SRM transmission becoming $T_{\mathrm{SRM}} \rightarrow 5 \%$.

The detuned configuration requires a more extreme adjustment with $T_{\mathrm{SRM}} \rightarrow 0.8 \%$. In addition, detuned operation results in imbalanced sidebands that not only impact the signal, but also the rf control fields used for alignment control and stabilizing internal degrees of freedom. Maintaining detuning using the current configuration of auxiliary fields requires adding control-point offsets, which can impact the reliability of continuous operation [30,31]. In total, detuning requires a considerable alteration of the operating controls and electronics, which would require significant time to implement.

\section{CONCLUSIONS}

Gravitational-wave detectors are currently configured to maximize the detectability of binary inspirals, rather than high-frequency signals. This leads to the question of whether existing interferometers can be tuned to target science at high frequencies, where binary neutron star postmerger waveforms contain information about the equation of state of dense nuclear matter. This work analyzed two approaches: wideband and detuned configurations. The wideband configuration provides a relative SNR improvement in detecting postmerger spectral peaks of $40-110 \%$ versus $\mathrm{A}+$.

Detuning LIGO's signal recycling cavity is another approach which maximizes sensitivity in a narrow band. For neutron star postmerger spectral peaks, detuning achieves an average SNR improvement between 0-40\% above the wideband configuration. Unlike the wideband configuration, the improvement from detuning is contingent on having empirical relations for the postmerger peak frequency and bandwidth as well as having the distributions of astrophysical parameters on which the relations depend. Both wideband and detuning configurations decrease the benefits imbued by squeezing by increasing the influence of interferometer loss, but only the detuned configuration diminishes squeezing by a significant amount. Detuning is also nontrivial to implement, and its relative SNR benefit does not appear sufficient to warrant trading implementation time for observing time, compared to the wideband configuration.

In the global network of gravitational-wave detectors, it could become advantageous to optimize one or more detectors for high-frequency postmerger signals. This work suggests that adjusting the interferometer bandwidth, rather than detuning, is the most promising avenue.

\section{ACKNOWLEDGMENTS}

The authors thank the National Science Foundation for support under Grant No. PHY-0555406. E. D. H. is supported by the MathWorks, Inc. The authors acknowledge fruitful discussions with Haixing Miao and Hartmut Grote in the early stages of this work, as well as valuable inputs from Stefan Ballmer, Teng Zhang and Peter Fritschel. Upper bounds on the SRC loss were based on data from Valera Frolov.

\section{APPENDIX: ASTROPHYSICAL DISTRIBUTIONS OF BNS POSTMERGER LORENTZIAN MODEL PARAMETERS}

This section establishes the phenomenological parameterizations used for the waveform template distributions. The form for the templates and their underlying phenomenological fits is derived from a set of numerical binary neutron star inspiral simulations [27]. The simulations and fits provide the general form for relating BNS system mass $M$ to the postmerger waveform central frequency, $f_{0}$. The cited work does not provide relations for the waveform $Q$, and this is discussed below.

In Sec. III the BNS postmerger signal was modeled as a Lorenztian with central frequency $f_{0}$ and quality factor $Q$. From Eq. (2), the peak frequency-domain strain amplitude for the Lorenztian is

$$
h_{\text {peak-f }}=\left|\tilde{h}_{\mathrm{DS}}\left(f_{0}\right)\right| \approx \sqrt{\frac{Q H}{\pi f_{0}}},
$$


which may be related to the peak strain in the time-domain waveform as

$$
h_{\text {peak-t }} \approx \frac{2 \pi f_{0}}{Q} h_{\text {peak-f }} .
$$

These peak strain formulas in time and frequency domains can be applied to Table I of [27] to derive the waveform $Q$ value for each numerical simulation.

Using the peak strain values and the $Q$, one can then determine the waveform signal energy, normalized by total mass and distance. The strain signal energy for a general template is

$$
H=\int_{-\infty}^{\infty}|h(t)|^{2} d t=\int_{-\infty}^{\infty}|\tilde{h}(f)|^{2} d f .
$$

For signals with a bandwidth small enough that the interferometer noise spectrum can be considered approximately constant in frequency, this expression leads to $\mathrm{SNR}^{2} \approx 4 H / \operatorname{PSD}\left(f_{0}\right)$. This approximation is why strain signal energy provides a particularly morphology independent SNR metric to be computed from numerical simulations. Additionally, $H$ can be related to the total energy emitted in the form of gravitational waves into the ringing postmerger signal. The energy in a strain signal is [7]

$$
E_{\mathrm{GW}}=\frac{c^{3}}{G} \frac{4}{5} \pi^{2} D^{2} \int_{-\infty}^{+\infty} f^{2}|\tilde{h}(f)|^{2} d f
$$

where $D$ is the distance to the source. This expression has an unphysical divergence if integrated to frequencies above $2 f_{0}$ for the damped-sine model. When the integral is confined to frequencies where $h_{\mathrm{DS}}$ is a good approximation, then in the limit $Q \gg 1$, the energy of a damped sine can be approximated as

$$
E_{\mathrm{GW}}=\frac{c^{3}}{G} \frac{4}{5} \pi^{2} D^{2} f_{0}^{2} H
$$

$M_{\mathrm{PM}}$ is the amount of mass that is converted to gravitational wave energy during the postmerger

$$
H=\left(2 \pi f_{0} D\right)^{-2} \frac{5 G}{c} M_{\mathrm{PM}}
$$

These waveform properties are used to formulate the dependence of the model templates on astrophysical parameters. The center frequency $f_{0}$ of the Lorentzian template model depends only on the total mass $M$ of the binary [28]:

$$
f_{0}(M, q)=\frac{C_{1}}{M}
$$

The constant $C_{1}$ parametrizes this dependence, and it is related to the tidal deformability constant $\tilde{\Lambda}$ of the binary. The distribution of $f_{0}$ then depends on the astrophysical distribution of masses of neutron stars in merging binary

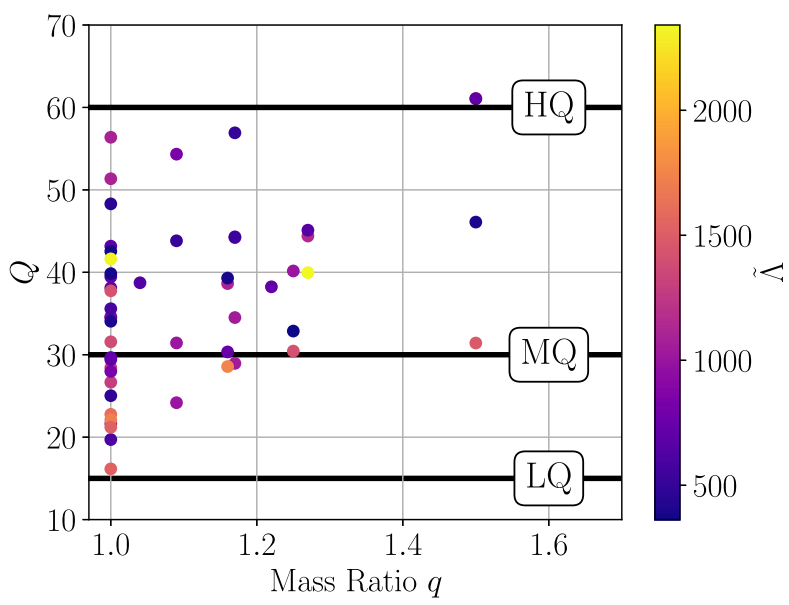

FIG. 4. Lorentzian $Q$ factors inferred from simulation data contained in Table I of [27] plotted against the mass ratio $q$ of the binary. The color bar shows the mass weighted tidal deformability $\tilde{\Lambda}$. The solid lines correspond to the values of $Q$ that have been chosen for the astrophysical distributions (see Table III) of Lorentzian signals in the analysis. These value are chosen in order to cover the range that is seen in simulation results.

systems. For this, we assume a Gaussian distribution of neutron star masses [32] centered around $1.35 M_{\odot}$ with a width of $0.05 M_{\odot}$.

In principle, the $Q$ is expected to depend on the binary's parameters, such as the mass ratio, the tidal deformability, and the equation of state. For a given post merger model, it could be assumed that the EoS and tidal deformability are constant, with the only important parameter varying astrophysically being the mass ratio $q$. Using Eq. (A2), Table I of [27] is used to calculate $Q$ and plot it against these parameters. Figure 4 shows that the dependence of $Q$ on the mass ratio $q$ does not appear to follow any particular functional form, but $Q$ lies between 15 and 60 for mass ratios $q<1$.6. In order to remain within this range, $Q$ is unlikely to be a strong function of $q$. Because the expected

TABLE III. Different astrophysical distributions for various choices of constants $C_{1}$ in Eq. (A7). The second last $M\left(f_{0}\right)$ represents the most likely $f_{0}$ for the distribution. Values of $C_{1}$ have been chosen in accordance with Table 1 of [28] to cover a range of frequencies (and tidal deformations). The distributions use fixed $Q$ s which have been chosen to cover the range of values that are obtained from simulation results (See Fig. 4).

\begin{tabular}{lccc}
\hline \hline Distribution & $C_{1}\left(\mathrm{kHz} M_{\odot}\right)$ & $M\left(f_{0}\right)(\mathrm{Hz})$ & $Q$ \\
\hline LQLF & 4.86 & 1800 & 15 \\
MQLF & 4.86 & 1800 & 30 \\
HQLF & 4.86 & 1800 & 60 \\
LQMF & 7.02 & 2600 & 15 \\
MQMF & 7.02 & 2600 & 30 \\
HQMF & 7.02 & 2600 & 60 \\
HQHF & 9.10 & 3333 & 60 \\
\hline \hline
\end{tabular}


mass ratio makes typical values of $q<1.2$, the astrophysical variability of $Q$ is sufficiently small that it does not affect this analysis, so it is fixed in each distribution. As a result, each distribution has only an astrophysical variation of $f_{0}$. The choice of constants, $C_{1}$ and $Q$ for each distribution is listed in Table III.
[1] B. Abbott et al., Phys. Rev. Lett. 119, 161101 (2017).

[2] B. P. Abbott et al., Astrophys. J. 848, L12 (2017).

[3] S. Bernuzzi, A. Nagar, M. Thierfelder, and B. Brügmann, Phys. Rev. D 86, 044030 (2012).

[4] T. Hinderer, B. D. Lackey, R. N. Lang, and J. S. Read, Phys. Rev. D 81, 123016 (2010).

[5] A. Bauswein and H.-T. Janka, Phys. Rev. Lett. 108, 011101 (2012).

[6] J. A. Clark, A. Bauswein, N. Stergioulas, and D. Shoemaker, Classical Quant. Grav. 33, 085003 (2016).

[7] K. Chatziioannou, J. A. Clark, A. Bauswein, M. Millhouse, T. B. Littenberg, and N. Cornish, Phys. Rev. D 96, 124035 (2017).

[8] H. Miao, H. Yang, and D. Martynov, Phys. Rev. D 98, 044044 (2018).

[9] D. Martynov, H. Miao, H. Yang, F. H. Vivanco, E. Thrane, R. Smith, P. Lasky, W. E. East, R. Adhikari, A. Bauswein, A. Brooks, Y. Chen, T. Corbitt, A. Freise, H. Grote, Y. Levin, C. Zhao, and A. Vecchio, Phys. Rev. D 99, 102004 (2019).

[10] K. Ackley et al., Pub. Astron. Soc. Aust. 37, e047 (2020).

[11] J. Miller, L. Barsotti, S. Vitale, P. Fritschel, M. Evans, and D. Sigg, Phys. Rev. D 91, 062005 (2015).

[12] L. Barsotti, L. McCuller, M. Evans, and P. Fritschel, The A+ design curve, Technical Report No. T1800042, LIGO, 2018.

[13] A. Buikema et al., Phys. Rev. D 102, 062003 (2020).

[14] A. Buonanno and Y. Chen, Phys. Rev. D 64, 042006 (2001).

[15] H. J. Kimble, Y. Levin, A. B. Matsko, K. S. Thorne, and S. P. Vyatchanin, Phys. Rev. D 65, 022002 (2001).

[16] M. Tse et al., Phys. Rev. Lett. 123, 231107 (2019).

[17] J. Mizuno, Comparison of optical configurations for laserinterferometric gravitational-wave detectors, Ph.D. thesis, Hannover University, 1995.

[18] J. Mizuno, K. A. Strain, P. G. Nelson, J. M. Chen, R. Schilling, A. Rüdiger, W. Winkler, and K. Danzmann, Phys. Lett. A 175, 273 (1993).

[19] E. Oelker, T. Isogai, J. Miller, M. Tse, L. Barsotti, N. Mavalvala, and M. Evans, Phys. Rev. Lett. 116, 041102 (2016).
[20] L. McCuller, C. Whittle, D. Ganapathy, K. Komori, M. Tse, A. Fernandez-Galiana, L. Barsotti, P. Fritschel, M. MacInnis, F. Matichard, K. Mason, N. Mavalvala, R. Mittleman, H. Yu, M.E. Zucker, and M. Evans, Phys. Rev. Lett. 124, 171102 (2020).

[21] M. Evans, L. Barsotti, P. Kwee, J. Harms, and H. Miao, Phys. Rev. D 88, 022002 (2013).

[22] A. Buonanno and Y. Chen, Classical Quant. Grav. 18, L95 (2001).

[23] A. Buonanno and Y. Chen, Phys. Rev. D 65, 042001 (2002).

[24] P. Fritschel, M. Evans, and V. Frolov, Opt. Express 22, 4224 (2014).

[25] T. T. Fricke, N. D. Smith-Lefebvre, R. Abbott, R. Adhikari, K. L. Dooley, M. Evans, P. Fritschel, V. V. Frolov, K. Kawabe, J. S. Kissel, B. J. J. Slagmolen, and S. J. Waldman, Classical Quant. Grav. 29, 065005 (2012).

[26] E. Capocasa, Y. Guo, M. Eisenmann, Y. Zhao, A. Tomura, K. Arai, Y. Aso, M. Marchiò, L. Pinard, P. Prat, K. Somiya, R. Schnabel, M. Tacca, R. Takahashi, D. Tatsumi, M. Leonardi, M. Barsuglia, and R. Flaminio, Phys. Rev. D 98, 022010 (2018).

[27] K. W. Tsang, T. Dietrich, and C. Van Den Broeck, Phys. Rev. D 100, 044047 (2019).

[28] S. Bernuzzi, T. Dietrich, and A. Nagar, Phys. Rev. Lett. 115, 091101 (2015).

[29] H. Miao, N. D. Smith, and M. Evans, Phys. Rev. X 9, 011053 (2019).

[30] O. Miyakawa, R. Ward, R. Adhikari, M. Evans, B. Abbott, R. Bork, D. Busby, J. Heefner, A. Ivanov, M. Smith, R. Taylor, S. Vass, A. Weinstein, M. Varvella, S. Kawamura, F. Kawazoe, S. Sakata, and C. Mow-Lowry, Phys. Rev. D 74, 022001 (2006).

[31] S. Hild, H. Grote, M. Hewtison, H. Lck, J. R. Smith, K. A. Strain, B. Willke, and K. Danzmann, Classical Quant. Grav. 24, 1513 (2007).

[32] F. Özel, D. Psaltis, R. Narayan, and A. Santos Villarreal, Astrophys. J. 757, 55 (2012). 